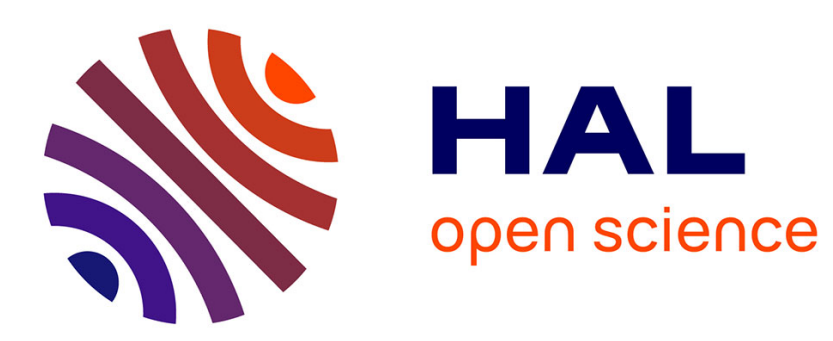

\title{
Active contour segmentation by use of a multichannel incoherent optical correlator
}

\author{
Eric Hueber, Laurent Bigue, Pierre Ambs
}

\section{To cite this version:}

Eric Hueber, Laurent Bigue, Pierre Ambs. Active contour segmentation by use of a multichannel incoherent optical correlator. Applied optics, 2003, 42 (23), pp.4681-4687. 10.1364/AO.42.004681. hal-00934615

\section{HAL Id: hal-00934615 \\ https://hal.science/hal-00934615}

Submitted on 22 Jan 2014

HAL is a multi-disciplinary open access archive for the deposit and dissemination of scientific research documents, whether they are published or not. The documents may come from teaching and research institutions in France or abroad, or from public or private research centers.
L'archive ouverte pluridisciplinaire HAL, est destinée au dépôt et à la diffusion de documents scientifiques de niveau recherche, publiés ou non, émanant des établissements d'enseignement et de recherche français ou étrangers, des laboratoires publics ou privés. 


\title{
Active contour segmentation by use of a multichannel incoherent optical correlator
}

\author{
Eric Hueber, Laurent Bigué, and Pierre Ambs
}

\begin{abstract}
We describe an optoelectronic incoherent multichannel processor that is able to segment an object in a real image. The process is based on an active contour algorithm that has been transposed to optical signal processing to accelerate image processing. This implementation requires exact-valued correlations and thus opens attractive perspectives in terms of optical analog computation. Furthermore, this optical multichannel processor setup encourages incoherent processing with high-resolution images. (C) 2003 Optical Society of America

OCIS codes: $\quad 070.0070,070.4550,070.5010,100.0100,100.1160$.
\end{abstract}

\section{Introduction}

Since the advent of the first optical convolvers and correlators, ${ }^{1-3}$ such processors have been used widely in optical pattern recognition (OPR) applications and are still at the cutting edge of technology according to the significant number of papers on OPR that appeared in leading optics journals over the last few years. OPR tasks usually detect, locate, recognize, and classify a target, by use of optical correlations. However, compared with the amount of work done in this field, another side of OPR has been quite eclipsed: the parametric description of the target.

Two descriptive operations have hardly been implemented in optics: the Hough transform ${ }^{4,5}$ and segmentation. The first provides information limited to objects that can be described with simple parametric curves (lines, ellipses, ....). As our objective is to describe real images containing more complicated targets or noisy environments, we dismissed this solution. The second is segmentation, which recently benefited from several improvements in digital implementations. The automatic extraction of objects in various types of images is a classic goal of image processing and a key operation in numerous pattern-recognition applications. However, only a

The authors are with ESSAIM (Ecole Supérieure des Sciences Appliquées pour l'Ingénieur-Mulhouse), Laboratoire Modélisation Intelligence Processus Systèmes, Université de Haute Alsace, 68 093 Mulhouse Cedex, France.

Received 8 November 2002; revised manuscript received 10 April 2003.

0003-6935/03/234681-07\$15.00/0

(C) 2003 Optical Society of America reduced number of optical processors operate with segmentation.

Michael and Arrathoon 6 have proposed optoelectronic image segmentation with logic arrays. On the contrary, Feng et al. ${ }^{7}$ have experimented with an analog optical computing solution. Their texturebased segmentation shows promising results. More recently, Barbé and Campos ${ }^{8}$ have developed an analog optical-segmentation processor that is restricted to objects textured with parallel lines.

No active contour segmentation has ever been implemented optically. The recent techniques with active contours named snakes ${ }^{9}$ consist in deforming a polygonal curve to fit the contour of the object to be segmented. Recently, new approaches to the snakebased segmentation of objects have been proposed. A technique called statistically independent region snake (SIRS) has been developed by Réfrégier et al. ${ }^{10,11}$ This method takes into account the statistical signature of regions to extract the object from the background. Numerical simulations have shown that SIRS snakes provide better results than common edge detectors in the presence of noise. ${ }^{12}$ These new segmentation algorithms can be expressed by use of correlation operations and consequently offer the possibility of being implemented with an optoelectronic architecture. Such a system seems attractive as far as speed is concerned. In Section 3 we propose a parallel processor to justify the optical implementation of this OPR operation.

Traditional optical correlators have always been dependent on the object to be located or detected. Moreover, their efficiency decreases drastically when the target becomes different from the reference. ${ }^{13,14}$ To reduce the dependence on the target attitude, it is 
possible to store different references of the target. ${ }^{15,16}$ In a different way, the optical processor described here performs segmentations with no prior knowledge of the target but the coordinates of one point belonging to the target region. The initial contour centered on this point adapts itself to the target's shape independently of its orientation and scale. This is why we find this implementation innovative and interesting, all the more so as it is based on a common OPR architecture. Its main interest is to enlarge the field of application for optoelectronic correlators.

A monochannel prototype processor with a shadowcasting incoherent correlator was previously implemented by our group. ${ }^{17}$ In this paper, we present an enhanced, higher-speed version with 4 channels.

The paper is organized as follows: The SIRS techniques are succinctly presented in Section 2 . Section 3 section deals with the choice and the conception of the optical parallel processor. Section 4 explains the iterative algorithm and Section 5 describes the simulations and experimental results of the optoelectronic SIRS snake processor.

\section{Active Contours}

Active contour segmentation provides more information than a simple segmentation operation: active contours not only give the binary separation between the object and background regions but also the coordinates of the contour vertices, the surface, the orientation and the position of the object. This is the main reason for the increasing interest in active contours during the last decade.

Active contour algorithms consist in minimizing a criterion iteratively to adapt the snake to the target's shape. While the first methods were based on energy minimization, statistical criteria have been introduced by Kervrann and Heitz. ${ }^{18}$ Such a framework is more adapted to real scene processings even if they are blurred or corrupted by noise. The SIRS model differentiates regions according to their surfaces, their variances, and the means of their histograms. Several algorithms are proposed in Ref. 11. They are adapted to images with different probability density functions (PDFs) that belong to the exponential family. These PDFs are defined by

$$
P^{\mu}(z)=k(z) \exp [\mathbf{a}(\mu) \beta(z)+f(\mu)]
$$

where $\mu=\left[\mu_{1}, \mu_{2}, \ldots, \mu_{n}\right]^{T}$ is the vector of the parameters of the PDF. $k(z)$ and the $p$ components of the vector $\beta(z)$ are functions of $z$ not depending on $\mu$, while the $p$ components of the vector $\mathbf{a}(\mu)$ and $f(\mu)$ are functions of $\mu$ not depending on $z$.

In this paper, the Gamma law ${ }^{19}$ layout for Gamma PDF is considered. This PDF characterizes images that can be segmented according to the means of the regions. The corresponding criterion, $J$, operates on the mean and surface of both the target and background regions. The criterion to minimize is then:

$$
\begin{aligned}
J(\mathbf{w}, \mathbf{s}) & =N(\mathbf{w}) \log (m)+[(N-N(\mathbf{w})] \log (\bar{m}) \\
m & =\frac{1}{N(\mathbf{w})}[\mathbf{s} \otimes \mathbf{w}]_{0} \\
\bar{m} & =\frac{1}{N-N(\mathbf{w})}\left(\sum_{i=1}^{N} s_{i}-[\mathbf{s} \otimes \mathbf{w}]_{0}\right),
\end{aligned}
$$

where $\mathbf{s}$ is the scene containing $N$ pixels; $\mathbf{w}$ stands for a binary window function that defines a shape for the target, so $\mathbf{w}$ is equal to 1 within this shape, and to 0 outside it. The purpose is to estimate the most likely shape for $\mathbf{w}$ for the target in the scene. $N(\mathbf{w})$ denotes the number of pixels equal to 1 in the window. The snake delimits the inner (target) and outer (background) regions. The 0 index associated with the correlation means that it stands for the central value of correlation.

Active contour is an adaptive operation that seems attractive for region tracking. However, the Gamma law criterion $J(\mathbf{w}, \mathbf{s})$ [Eqs. (2)-(4)] contains one correlation. Such an operation may require significant time and depends on the scene size. An optical processor is therefore proposed to compute this algorithm benefit from the advantages of optics. More precisely, the field of application for optical correlators that are capable to locate and track a target of known shape is thus enlarged to the new capability of segmenting a target of unknown shape but approximately known location.

\section{Optical Implementation}

The optical correlation is computed by an incoherent correlator based on the shadow-casting principle. ${ }^{20,21}$ We already validated this architecture ${ }^{17}$ to process optical segmentations. The purpose of this paper is to describe how our prototype has been improved by taking account of the parallel processing capabilities inherent in incoherent optical processors. ${ }^{22}$ The major drawback of this architecture is the slight diffraction of the first plane that is largely compensated by three fundamental advantages. First, it is not corrupted by coherent noises like speckle. Second, in a coherent light processor, twisted nematic liquid crystal spatial light modulator(s) (SLM), such as the ones used for this study, couple amplitude with phase. A shadow-casting incoherent correlator operates with intensity values and therefore perturbations due to phase-amplitude coupling can be neglected. ${ }^{23}$ Third, a processor with an incoherent light source is hardly sensitive to optical surface irregularities whereas the performance of traditional optical correlators drops drastically in the presence of such irregularities. Moreover, it benefits from a weak sensitivity to misalignment.

The optical design (Fig. 1) features the detection plane $P_{\otimes}$ that is located in front of $P_{w}$ and $P_{s}$. Therefore a lens is necessary to image the correlation, $P_{\otimes}$, 


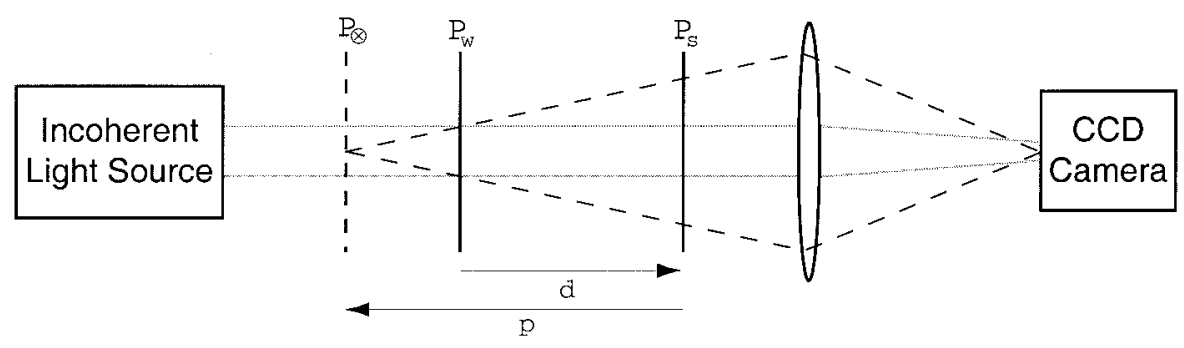

Fig. 1. Configuration of the optical processor. Plane $P_{\otimes}$ contains the correlation of the amplitude distributions located in planes $P_{\mathrm{W}}$ and $P_{\mathrm{S}}$.

in the camera plane. The multichannel optical processor that operates the correlation between scene $\mathbf{s}$ and window $\mathbf{w}$ is schematized in Fig. 2.

As shown in Fig. 2, two achromats image the demagnified correlation plane onto the CCD array. The diagonal of both SLMs used is $33 \mathrm{~mm}$. The diagonal of the CCD array is $8 \mathrm{~mm}$. Thus fourfold demagnification is required. If only one achromat was used, an $F$-number should be lower than one. This is due to the fact that vignetting must be avoided. ${ }^{24}$

The light source used here consists of a laser source associated with a rotating diffuser. Indeed, it is the best light source for incoherent light processes according to Ref. 25. The coherent light is first collimated and then goes through the rotating diffuser. The resulting beam is Gaussian and incoherent. It illuminates a SVGA (i.e., super video graphics array with $800 \times 600$ pixels) SLM where the multiplexed window $\mathbf{w}$ is displayed. Then, the scene shadow is cast on an SVGA SLM where the scene $\mathbf{s}$ is displayed. In the following, we refer to the scene SLM as $\mathrm{SLM}_{\mathrm{S}}$ and to the window SLM as $\mathrm{SLM}_{\mathrm{W}}$. Eventually, the two achromats image $P_{\otimes}$ on the CCD camera. As the measurements in incoherent light are related to amplitude and not to intensity, the camera directly acquires the correlation product of $\mathbf{s}$ by $\mathbf{w}\left(P_{\otimes}\right)$. The criterion computation only requires the correlation center that is blurred because of the diffraction effect. ${ }^{26}$ Consequently, the central value of the correlation acquired by this analog optical system must be averaged on a few CCD cells instead of a unique one.

The resolution of incoherent optical correlators are limited by the diffraction effect. The light passing through $\mathrm{SLM}_{\mathrm{W}}$ is diffracted by $\mathrm{SLM}_{\mathrm{S}}$. Green ${ }^{27}$ stud- ied the maximum resolution achievable with a shadow-casting system. The following equation can be applied to our system to approximate the resolution cells: 28

$$
N_{\mathrm{W}} N_{\mathrm{S}}=\frac{A_{\mathrm{W}} A_{\mathrm{S}}}{4 \alpha^{2} \lambda^{2} d^{2}}
$$

where $A_{\mathrm{W}}$ and $A_{\mathrm{S}}$ correspond to the total surface planes used by the SLM aperture $\left(26 \times 20 \mathrm{~mm}^{2}\right)$. Assuming that the number of resolution cells in the window and the scene are equal $\left(N_{\mathrm{W}}=N_{\mathrm{S}}\right)$, for a distance $d=119 \mathrm{~mm}$ between the SLM, an illumination wavelength $\lambda=532 \mathrm{~nm}$, and a pixel size of 33 $\mu \mathrm{m}$, correlation of images with space-bandwidth product

$$
N_{\mathrm{W}}=N_{\mathrm{S}} \approx 72 \times 94,
$$

may be obtained without any loss in resolution due to diffraction. For these numbers, the diagonal of each pixel is $\sim 390 \mu \mathrm{m}$ that corresponds to approximately 72 pixels. We must be aware that the resolution of shadow-casting systems given by expression (5) is only approximative. A more accurate diffraction analysis can be found in Ref. 26 . In fact, we attempted to override this limitation and thus process images supposed to be subject to diffraction.

Additionally, photometric effects specific to the incoherent light architecture and the Gaussian profile of the laser beam affect spatially the intensity of the light beam. ${ }^{24}$ So, the farther the data are from the optical axis, the less energetic they are. This is why we added a second optical branch: to correct the beam energy delivered by the output of the first SLM containing the multiplexed window and to make it

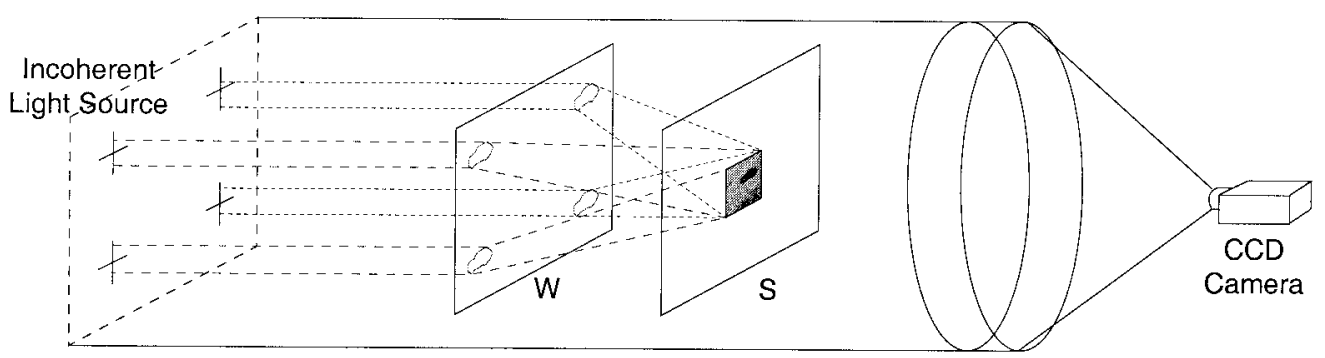

Fig. 2. Multichannel incoherent shadow-casting correlator with four channels. 


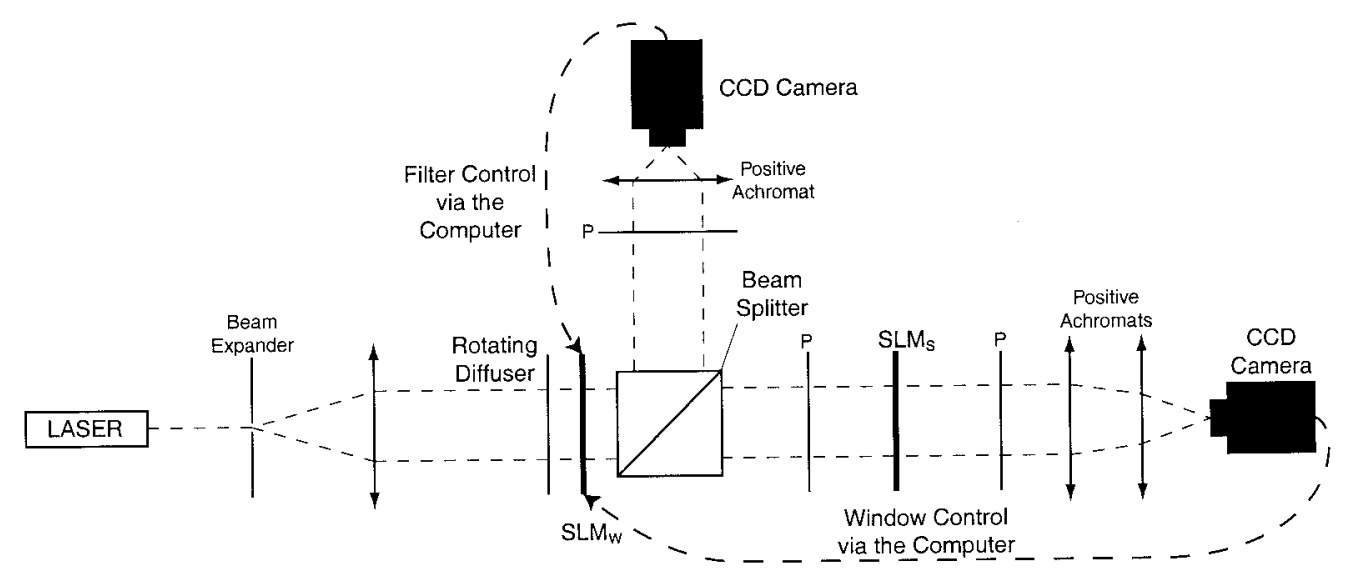

Fig. 3. Complete optical setup of the multichannel incoherent correlator.

uniform. The complete setup of our system is represented in Fig. 3.

Because there were slight diffraction and photometric effects in the monochannel configuration, they have to be corrected or taken into account to perform efficient segmentations with our multichannel setup.

The results are also improved by use of basic numerical operations, such as contrast enhancement and occasionally contrast reversal to maximize the energy in the target region.

\section{Convergence Strategy}

To improve the speed of our system restrained, for the moment, by the SLMs and the cameras, we added three new processing optical channels thus optimizing the available surface. Several strategies can be investigated with the multichannel configuration. Most of them concern quality improvement, but our goal is to accelerate the process. So, we opted for a solution where each parallel channel correlates simultaneously a different deformation of the binary window with the unique scene. Each window differs with displacement of one different node moved in a stochastic direction. Whereas iterations were oper- ated serially with the computer or the monochannel configuration, they now benefit from the optical parallel processing.

For instance, the strategy applied to our fourchannel correlator and eight-node snake (Fig. 4) follows this iterative algorithm:

1. Initialize the contour and compute optically the corresponding criterion in each channel.

2. Displace stochastically four nodes (one node per channel).

3. Compute optoelectronically the criterion for each channel (i.e., four criteria).

4. Validate each deformation minimizing the criterion of the previous iteration (i.e., the deformation that brought the active contour closer to the edge of the area of interest).

5. Go back to Step 2 or stop the iterative process after 50 iterations.

As there are eight nodes, the contour is entirely deformed with two four-channel correlations. The convergence algorithm is stopped after 50 iterations because no significant improvement is obtained with further steps.

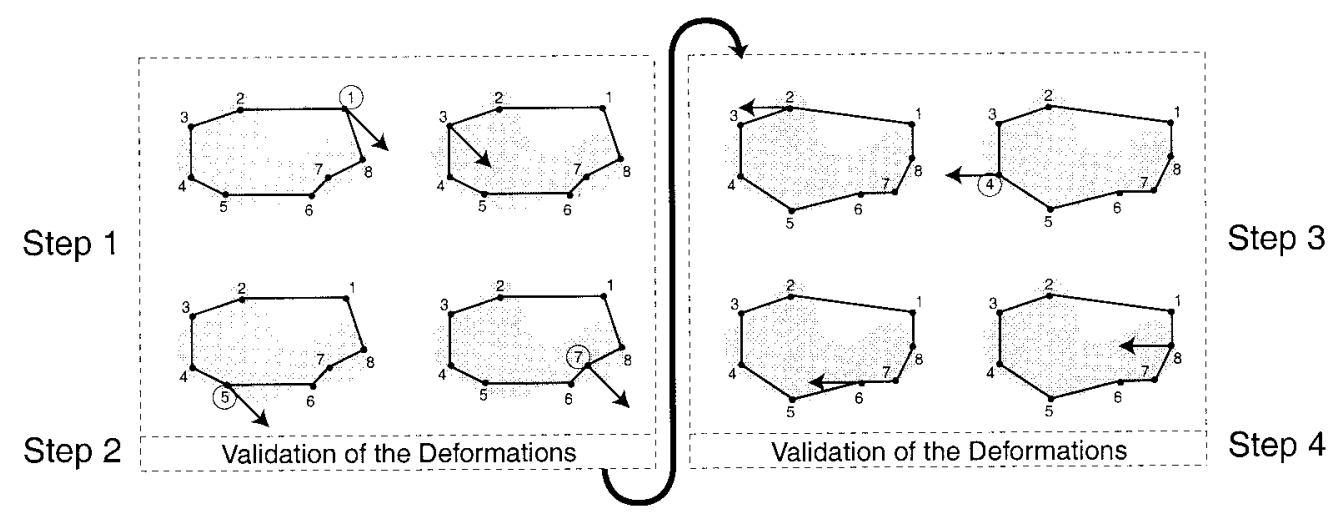

Fig. 4. Strategy applied to the four-channel correlator. Step 1: Odd numbered nodes are moved in the same stochastic direction. Step 2: Deformations that minimize the criterion (nodes 1, 5, and 7) are applied to obtain the new window. The new binary window is displayed in the four channels to measure their criterion. Step 3: Even numbered nodes are moved in a new stochastic direction. Step 4: Same as Step 2 with the validation of the deformation of node number 4 . 

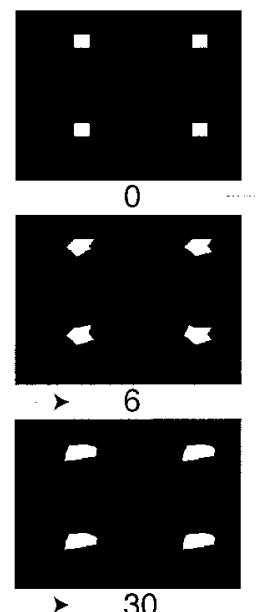
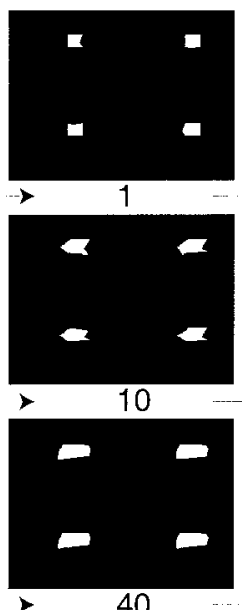

(a)

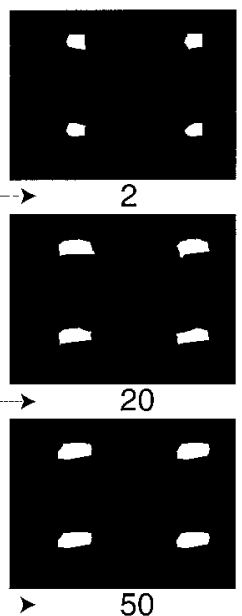

50

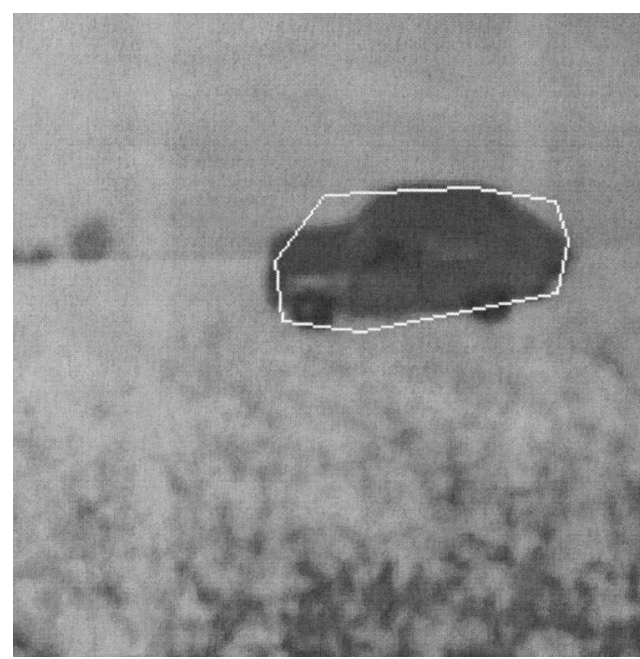

(b)

Fig. 5. (a) Several multiplexed windows extracted from the 50-iteration segmentation, (b) final contour obtained with the optical processor superimposed on the original scene.

\section{Experimental Results}

Gamma law segmentation is applied to $249 \times 249$ images. The scene shows a car in a field. To demonstrate that the processor is efficient for real images, the photo is slightly defocused and badly contrasted. Some multiplexed windows of the 50-iteration segmentation process are presented in Fig. 5 to show how the optoelectronic SIRS snake behaves.

To evaluate the quality of segmentation, we had to choose a method for objective evaluation. It is very difficult to find a single quantitative index for this purpose because such an index should take into account many factors, such as homogeneity, contrast, compactness, continuity, psycho-visual perception, etc. A human being is possibly the best judge for this. Anyway, one of the best automatic methods is based on the Hausdorff distances. ${ }^{29}$ This technique might appear more complicated than another, slightly less accurate, but so much more simpler solution that consists in an XOR (i.e., exclusive OR) Boolean operation between the surface obtained through segmentation and a reference image. Our synthetic reference results from an average between several numerical segmentations. The quality is then:

$$
Q=W \oplus W_{\text {Ref }}
$$

Obviously, quality quantification can only be operated with a known scene. The quality of this method depends on the accuracy of the reference.

The experimental optoelectronic results shown in Fig. 5 were obtained after 50 iterations for a quality of $\sim 82 \%$, whereas simulation with 8 nodes were used to reach $88 \%$. This difference lies in the fact that the analogic system is subject to temporal perturbations introduced by the rotating nonhomogeneous diffuser, the SLM flicker, and spatial disturbances due to diffraction and photometry effects.
The algorithm can be optimized to process highquality segmentation with many nodes and iterations. From an experimental point of view, a progressive increase in the number of nodes does not really improve segmentation accuracy. Indeed, the more nodes the active contour contains, the more sensitive it is to noise. For the scene studied in this paper, a contour of eight nodes seems to be the best choice because more nodes do not really improve the quality and fewer nodes deliver results that are too rough.

Nodes are moved stochastically, so the results may occasionally be slightly different. Indeed, the active contour that works in a noisy environment might converge toward several different local minima. Thus the segmentation results may not be the only one. This is valid for both experimental and simulation results. Therefore the experimental results shown in Fig. 5 can be regarded as satisfactory.

As demonstrated previously, our optical snakebased processor is also effective with a further overlapping white Gaussian noise. ${ }^{17}$ The proposed multichannel implementation also inherits the noise robustness of the SIRS model.

The optical segmentation speed is limited (presently, with a twisted nematic liquid crystal SLM, it is equal to $30 \mathrm{~s}$ with the optical processor) first, by the refresh rate of the SLMs and second, by the camera acquisition time. Recently, our group acquired a new ferroelectric SLM with gray-level capabilities. ${ }^{30,31}$ The device features a refresh rate of approximately one kilohertz and may provide much faster segmentations. Besides, the camera that is the other speed reducer can be replaced by fast photodetectors, or by a high-speed CCD, or complementary metal-oxide semiconductor devices, because only the central value of the correlation is acquired. All these improvements with the multichannel setup are 
meant to reduce segmentation time that will tend toward a tenth of a second.

The location of a target of known shape and the segmentation of an approximately located target are the two fundamental operations in OPR. These two aspects can be associated to perform enhanced optical operations, such as in Ref. 32 where a preliminary optical snake-based segmentation eliminates the background and thus improves the correlation-based optical target tracking.

\section{Conclusion}

We have designed and implemented a shadowcasting incoherent multichannel correlator that is able to segment images using an active contour. Because this system works with wide data planes, we applied an adaptive beam homogenization. The multichannel setup we proposed performs segmentation twice as fast as our previous monochannel system. We obtained satisfactory experimental results even if the theoretical resolution limitations due to diffraction were overstepped. Such incoherent processors may thus look more attractive.

This processor gives the contour of an object starting from a point belonging to its inner region. In a way, it is the opposite operation of a traditional correlator that finds the position of the object, knowing its shape. Moreover, the statistical framework provided by the SIRS algorithms improves the noise robustness of the system. Thus this experiment opens new possible applications for classic optical correlation architectures. For instance, our multichannel processor is able to operate exact-valued correlations in parallel and is therefore attractive as an optical calculator. Eventually, the promising results may lead to a real-time solution. Scene preprocessing, which is required in many cases, will benefit from the optical design. An optoelectronic architecture that can perform detection, tracking, estimation, and segmentation, and thus enable efficient target tracking or recognition in real time (i.e., at a video frame rate), is now conceivable.

The authors wish to thank Philippe Réfrégier for his fruitful comments and help.

\section{References}

1. W. Meyer-Eppler, "Die funktionalanalytische Behandlung des Schattenproblems," Optik (Stuttgart) 1, 465-474 (1946).

2. F. B. Berger, "Optical cross-correlator," U.S. patent $2,787,188$ (1957).

3. A. VanderLugt, "Signal detection by complex spatial filtering," IEEE Trans. Inf. Theory 10, 139-145 (1964).

4. P. V. C. Hough, "Method and means for recognizing patterns," U.S. patent 3,069,654 (1962).

5. S. Laut, F. Xu, P. Ambs, and Y. Fainman, "A matrix of $64 \times 64$ computer generated holograms for an optical Hough transform processor," in Optical Information Science and Technology, (OIST 97): Optical Theory and Neural Networks, A. Mikaelian, ed., Proc. SPIE 3402, 22-31 (1998).

6. N. Michael and R. Arrathoon, "Optoelectronic parallel watershed implementation for segmentation of magnetic resonance brain images,” Appl. Opt. 36, 9269-9286 (1997).

7. W. Feng, Y. Yan, G. Huang, and G. Jin, "Micro-optical multi- wavelet element for hybrid texture segmentation processor," Opt. Eng. 37, 185-188 (1998).

8. J. Barbé and J. Campos, "Image segmentation with a white light optical correlator," in 18th Congress of the International Commission for Optics, San Francisco, A. J. Glass, J. W. Goodman, M. Chang, A. H. Guenther, and T. Asakura, eds., Proc. SPIE 3749, 775-776 (1999).

9. M. Kass, A. Witkin, and D. Terzopoulos, "Snakes: active contour models," Int. J. Comput. Vision 1, 321-331 (1988).

10. O. Germain and P. Réfrégier, "Optimal snake-based segmentation of a random luminance target on a spatially disjoint background," Opt. Lett. 21, 1845-1847 (1996).

11. C. Chesnaud, P. Réfrégier, and V. Boulet, "Statistical region snake-based segmentation adapted to different physical noise models," IEEE Trans. Pattern Anal. Mach. Intell. 21, 11451157 (1999).

12. O. Germain, "Segmentation d'images radar: caractérisation des détecteurs de bord et apport des contours actifs," Ph.D. dissertation, University of Aix-Marseille III, France (2001).

13. J. L. Horner and P. Gianino, "Additional properties of the phase-only correlation filter," Opt. Eng. 23, 695-697 (1984).

14. Q. Tang and B. Javidi, "Sensitivity of the nonlinear joint transform correlator: experimental investigation," Appl. Opt. 31, 4016-4024 (1992).

15. B. V. K. Vijaya Kumar, "Tutorial survey of composite filter designs for optical correlators," Appl. Opt. 31, 4773-4801 (1992).

16. L. Bigué, M. Fracès, and P. Ambs, "Experimental implementation of a joint transform correlator using synthetic discriminant functions," Opt. Las. Eng. 23, 93-111 (1995).

17. E. Hueber, L. Bigué, P. Réfrégier, and P. Ambs, "Optical snake-based segmentation processor with a shadow-casting correlator," Opt. Lett. 26, 1852-1854 (2001).

18. C. Kervrann and F. Heitz, "Robust tracking of stochastic deformable models in long image sequences," in Proceedings of IEEE Conference on Computer Vision Pattern Recognition (Seattle, 1994), pp. 724-728.

19. P. Réfrégier, O. Germain, and T. Gaidon, "Optimal snake segmentation of target and background with independent Gamma density probabilities, application to speckled and preprocessed images," Opt. Commun. 137, 382-388 (1997).

20. M. M. Robertson, "Interpretation of Patterson diagrams," Nature 152, 411-412 (1943).

21. L. Bragg, "Lightning calculations with light," Nature 154, 69-72 (1944).

22. G. L. Rogers, Noncoherent Optical Processing (Wiley, New York, 1977).

23. V. Laude, "Corrélation optique optimale et application aux architectures cohérentes et incohérentes," $\mathrm{Ph} . \mathrm{D}$. dissertation, University of Paris XI Orsay (1994).

24. M. Gedziorowski and J. Garcia, "Programmable opticaldigital processor for rank order and morphological filtering," Opt. Commun. 119, 207-217 (1995).

25. D. P. Casasent and G. House, "Implementation issues for a noncoherent optical correlator," in Optical Pattern Recognition V, D. P. Casasent and T.-H. Chao, eds., Proc. SPIE 2237, 179-188 (1994).

26. V. Laude, "Diffraction analysis of pixelated incoherent shadow casting," Opt. Commun. 138, 394-402 (1997).

27. E. L. Green, "Diffraction in lensless correlation," Appl. Opt. 7, 1237-1239 (1968).

28. V. Laude, P. Chavel, and P. Réfrégier, "Implementation of arbitrary real-valued correlation filters for the shadow-casting incoherent correlator,” Appl. Opt. 35, 5267-5274 (1996).

29. O. Ruch and P. Réfrégier, "Comparison of Hausdorff distances performance in dissimilarity measurements for silhouette dis- 
crimination," in Automatic Target Recognition XI, F. A. Sadjadi, ed., Proc. SPIE 4379, 454-465 (2001).

30. K. A. Bauchert and S. A. Serati, "High-speed multi-level $512 \times$ 512 spatial light modulator," in Optical Pattern Recognition XI, D. P. Casasent and T.-H. Chao, eds., Proc. SPIE 4043, 59-65 (2000).

31. P. Ambs and L. Bigué, "Characterization of an analog ferroelectric spatial light modulator. Application to dynamic diffractive optical elements and optical information processing," in Optoelectronic Information Processing: Optics for Information Systems, 4th Euro-American workshop on optoelectronic Information Processing, P. Réfrégier, B. Javidi, C. Ferreira, and S. Vallmitjana, eds., SPIE Critical Review CR81, 365-393 (2001).

32. A. Matwyschuk, P. Ambs, and F. Christnacher, "Target tracking correlator assisted by a snake-based optical segmentation method," Opt. Commun. 219, 125-137 (2003). 